\title{
A new approach to analyticity of Dirichlet-Neumann operators
}

\author{
David P. Nicholls and Fernando Reitich \\ School of Mathematics, University of Minnesota, \\ Minneapolis, MN 55455, USA
}

(MS received 19 October 1999; accepted 2 February 2001)

\begin{abstract}
This paper outlines the theoretical background of a new approach towards an accurate and well-conditioned perturbative calculation of Dirichlet-Neumann operators (DNOs) on domains that are perturbations of simple geometries. Previous work on the analyticity of DNOs has produced formulae that, as we have found, are very ill-conditioned. We show how a simple change of variables can lead to recursions that satisfy analyticity estimates without relying on subtle cancellation properties at the heart of previous formulae.
\end{abstract}

\section{Introduction}

In many problems of physical relevance one must solve complicated systems of partial differential equations on perturbations of simple domains. In this situation, Dirichlet-Neumann operators (DNOs) can play an important role, especially if information at the boundary is all that is required. Often, systems of partial differential equations formulated on the entire domain can be reduced to equations defined only at the boundary by using DNOs. This is the case, for instance, for a variety of free boundary problems from fluid mechanics (water waves, Hele-Shaw flows, etc. [19]) and solid-state physics (Stefan problems, crystal growth, etc. [15]). Alternatively, DNOs may serve computational purposes, such as in the realization of radiation conditions in scattering problems (see $[16,17]$ and the references therein). If the domain deviates from an exactly solvable geometry, then a perturbative estimation of DNOs can yield excellent results $[10,11,20,21]$. In this context, a detailed understanding of analyticity properties of DNOs is vital to determining the range of applicability of such approximations.

In this paper we develop the theoretical background of a new approach towards an accurate and well-conditioned perturbative calculation of DNOs on domains that are perturbations of simple geometries. As a byproduct, we produce a novel proof of analyticity of DNOs associated with Laplace's equation upon boundary perturbations. Previous results on analyticity properties of DNOs include those of Coifman and Meyer [8], who considered small Lipschitz perturbations of a line in the plane, and those of Craig et al. [12] and Craig and Nicholls [9] on $C^{1}$ perturbations of a hyperplane in three and $n$ dimensions, respectively. In every case, the derivation of such results relied on subtle estimates of the associated singular integral operators. In contrast, our own approach, applicable to somewhat smoother perturbations (slightly less than $C^{2}$ ), is based on classical and explicit estimation of recursions 
that result from the exact solvability of Laplace's equation on a strip (or in a halfspace).

In fact, a different version of these recursions had been previously derived and numerically implemented by Craig and Sulem [11], Schanz [21] and Nicholls and Craig $[10,20]$. Our main observation relates to the subtle cancellations present in these formulae that manifest themselves as severe numerical ill-conditioning (see $\S 3$ ). Our resolution of this issue is accomplished, quite simply, by a change of variables (see $\S 4$ ). Indeed, as we shall show, a transformation of the varying domains onto the fixed unperturbed geometry bypasses these cancellations without compromising the recursive nature of the formulae. In this manner, and in contrast with the original formulation, the estimation of the solution of the successive problems can proceed without regard for the interactions between previously computed terms (see $\S 5$ ). More important, perhaps, are the possible numerical implications of this approach. Our convergence proof suggests that no significant cancellation occurs in our new recursive formulae, which should, therefore, prove numerically more stable. Preliminary results do indeed confirm this hypothesis; a thorough investigation of the corresponding numerical method and other algorithmic issues, however, will be left for future work.

\section{Dirichlet-Neumann operators}

\subsection{Motivation and definition}

The relevance of DNOs goes beyond their mathematical interest, as they have become an integral part of simulation models in a variety of areas. In this section we motivate their study within the context of the classical free boundary model of gravity water waves. As will be clear from the discussion, an analogous treatment is possible for other free boundary models such as Stefan, Hele-Shaw or MullinsSekerka problems. The model we shall consider assumes an ocean of finite or infinite depth with periodic surface waves. For the sake of definiteness, we shall perform our analysis of DNOs for such configurations. Extensions to perturbations from other separable geometries are immediate.

The classical problem of water waves [19] relates to the motion of an ideal (inviscid, irrotational and incompressible) fluid under the influence of gravity. The fluid occupies the region

$$
S_{h, \eta}=\left\{(x, y) \in \mathbb{R}^{N-1} \times \mathbb{R} \mid-h \leqslant y \leqslant \eta(x, t)\right\},
$$

where $h$ is the depth (possibly infinite) and $\eta$ specifies the water surface. In the case of finite depth, no generality is lost by setting $h=1$, as this amounts to a simple rescaling of independent variables. We adopt this convention and note that any information concerning $h$ dependence of the constants in our results can be recovered a posteriori using a scaling argument. Thus the fluid motion is governed by [19]

$$
\begin{array}{rlrl}
\Delta \varphi & =0 & & \text { in } S_{1, \eta}, \\
\partial_{y} \varphi=0 & & \text { at } y=-1, \\
\partial_{t} \eta+\nabla_{x} \varphi \cdot \nabla_{x} \eta-\partial_{y} \varphi & =0 & & \text { at } y=\eta, \\
\partial_{t} \varphi+\frac{1}{2}|\nabla \varphi|^{2}+g \eta & =0 & & \text { at } y=\eta,
\end{array}
$$


where $\varphi=\varphi(x, y, t)$ is the velocity potential and $g$ is the acceleration due to gravity. To consider water of infinite depth, equation $(2.1 b)$ is replaced with the condition

$$
\nabla \varphi \rightarrow 0 \quad \text { as } y \rightarrow-\infty
$$

To complete the description of the fluid's evolution, boundary conditions in the horizontal direction must be imposed. As in many classical treatments [19], we assume periodicity of the structure: given a lattice $\Gamma$ and a parallelogram $P(\Gamma) \subset \mathbb{R}^{N-1}$ based on that lattice, we require that $\varphi(x+\gamma, y, t)=\varphi(x, y, t)$ and $\eta(x+\gamma, t)=\eta(x, t)$ for all $\gamma \in \Gamma$.

As pointed out by Zakharov [23] and Craig and Sulem [11], for both theoretical and computational analysis, a convenient form of the water waves problem can be achieved through its reformulation in the form of a Hamiltonian system. This, however, requires the introduction of DNOs associated with Laplace's equation on the domain $S_{1, \eta}$. As their names suggest, DNOs take Dirichlet data at the surface and return Neumann data. More precisely, within the present context, they can be defined as follows.

Definition 2.1. Consider the domain

$$
S_{\sigma}=\{(x, y) \in P(\Gamma) \times \mathbb{R} \mid-1 \leqslant y \leqslant \sigma(x)\} .
$$

Given Dirichlet data $\xi(x)$, the unique solution $v(x, y)$ of

$$
\begin{array}{rlrl}
\Delta v & =0 \quad & \\
v(x, \sigma(x)) & =\xi(x) & \\
\partial_{y} v(x,-1) & =0 & \\
v(x+\gamma, y) & =v(x, y) \quad \text { for all } \gamma \in \Gamma
\end{array}
$$

can be found and the Dirichlet-Neumann operator $G(\sigma)$ is defined as

$$
G(\sigma) \xi=\left.\nabla v\right|_{y=\sigma} \cdot N_{\sigma}
$$

where

$$
N_{\sigma}=\left(-\nabla_{x} \sigma, 1\right)^{\mathrm{T}}
$$

Once again, equation $(2.2 c)$ should be replaced by

$$
\nabla v \rightarrow 0 \quad \text { as } y \rightarrow-\infty
$$

in the case of infinite depth.

With this definition, the Hamiltonian of the water wave problem is

$$
H(\eta, \xi)=\frac{1}{2} \int_{P(\Gamma)} \xi G(\eta) \xi+g \eta^{2} \mathrm{~d} x
$$

and the equations of motion can be posed entirely at the free surface,

$$
\partial_{t} \eta=\delta_{\xi} H, \quad \partial_{t} \xi=-\delta_{\eta} H,
$$

where $\xi(x, t)=\varphi(x, \eta(x, t), t)[11,23]$. 


\subsection{Analytic dependence on perturbations}

For simple separable geometries, DNOs can be easily computed. For instance, if $\sigma=0, h=\infty$ and $\Gamma^{\prime}$ denotes the lattice conjugate to $\Gamma$, then

$$
G(0)\left[\sum_{k \in \Gamma^{\prime}} \hat{\xi}(k) \mathrm{e}^{\mathrm{i} k \cdot x}\right]=\sum_{k \in \Gamma^{\prime}}|k| \hat{\xi}(k) \mathrm{e}^{\mathrm{i} k \cdot x},
$$

that is, $G(0)=H \nabla_{x}$, where $H$ denotes the Hilbert transform. On the other hand, for general surfaces, the numerical estimation of DNOs presents substantial challenges (see, for example, $[1,2]$ and the references therein). For small deviations of a hyperplane (or other exactly solvable configurations), however, a perturbative method offers an appealing approach [10,11,20,21]. Indeed, if

$$
\sigma(x)=\varepsilon f(x)
$$

for $\varepsilon \ll 1$, then a formal approximation of $G(\sigma)$ in the form of a power series in $\varepsilon$ can be derived. Indeed, letting $v=v(x, y, \varepsilon)$ denote the solution of

$$
\begin{aligned}
\Delta v & =0 \quad \text { in } S_{\varepsilon f}, \\
v(x, \varepsilon f, \varepsilon) & =\xi(x), \\
\partial_{y} v(x,-1) & =0, \\
v(x+\gamma, y) & =v(x, y) \quad \text { for all } \gamma \in \Gamma,
\end{aligned}
$$

then

$$
G(\varepsilon f) \xi=\partial_{y} v-\varepsilon \nabla_{x} f \cdot \nabla_{x} v .
$$

To find the series expansion

$$
G(\varepsilon f) \xi=\sum_{n=0}^{\infty}\left(G_{n}(f) \xi\right) \varepsilon^{n}
$$

we note $[13]$ (see also [4-6]) that the expansion for $v$,

$$
v(x, y, \varepsilon)=\sum_{n=0}^{\infty} v_{n}(x, y) \varepsilon^{n},
$$

can be recursively computed by solving

$$
\begin{array}{rlrl}
\Delta v_{n} & =0 & & \text { in } S_{0}, \\
v_{n}(x, 0) & =H_{n}(x), & \\
\partial_{y} v_{n}(x,-1) & =0, \\
v_{n}(x+\gamma, y) & =v_{n}(x, y) \quad \text { for all } \gamma \in \Gamma,
\end{array}
$$

where

$$
H_{n}(x)=-\sum_{l=0}^{n-1} \frac{f^{n-l}}{(n-l) !} \partial_{y}^{n-l} v_{l}(x, 0)+\delta_{n, 0} \xi(x)
$$

and $\delta_{j, k}$ is the Kronecker delta. 
The validity of such an approach hinges, of course, on the plausibility of the expansion (2.4), that is, on the analyticity properties of DNOs upon boundary variations. Results to this effect for Lipschitz perturbations of a line in two dimensions follow from the work of Calderón [7] and Coifman and Meyer [8]. More specifically, for a Lipschitz profile $f$, a delicate analysis of the associated singular integral operators can be used to derive estimates for $G_{n}$ in $(2.4)$ of the form

$$
\left\|G_{n}(f) \xi\right\|_{L^{2}} \leqslant C B^{n}\|\xi\|_{H^{1}}
$$

where $B$ depends on the $L^{\infty}$ norm of $f^{\prime}$. Similar results for $C^{1}$ perturbations of hyperplanes in three and more dimensions have been recently established in [9,12].

While these results establish the convergence of the expansion (2.4), the interpretation of the recursion (2.6) demands more careful attention. A close inspection of these formulae would appear to indicate a requirement that the boundary surface $y=f(x)$ be infinitely differentiable and, indeed, under the assumption of its spatial analyticity, the explicit relations (2.6) have been shown to hold [3]. For general Lipschitz perturbations, on the other hand, the individual terms in the right-hand side of (2.6) may not be well defined and the overall validity of the formulae arises from certain cancellations that give meaning to these equations. This observation, and its potential numerical implications ( see $\S 3$ ), becomes even clearer perhaps as we consider an alternative algorithm for the computation of DNOs that only involves the Taylor coefficients $G_{n}(f)[11]$.

Following [11], we fix $p \in \Gamma^{\prime}$ and consider

$$
w_{p}(x, y)=\mathrm{e}^{\mathrm{i} p \cdot x+|p| y},
$$

a solution of $(2.2 a),(2.2 d)$ and (2.3). If $\sigma=\varepsilon f$, then

$$
w_{p}(x, \varepsilon f)=\mathrm{e}^{\mathrm{i} p \cdot x+|p| \varepsilon f},
$$

so that

$$
G(\varepsilon f)\left[\mathrm{e}^{\mathrm{i} p x+|p| \varepsilon f}\right]=\left.\left(\partial_{y}-\varepsilon \nabla_{x} f \cdot \nabla_{x}\right)\left(\mathrm{e}^{\mathrm{i} p \cdot x+|p| y}\right)\right|_{y=\varepsilon f},
$$

that is,

$$
G(\varepsilon f)\left[\mathrm{e}^{\mathrm{i} p x+|p| \varepsilon f}\right]=\left(|p|-\varepsilon \nabla_{x} f \cdot \mathrm{i} p\right) \mathrm{e}^{\mathrm{i} p \cdot x+|p| \varepsilon f} .
$$

Thus, expanding the equality (2.8) in the form of a series in $\varepsilon$ and equating like powers, we obtain

$$
\begin{aligned}
G_{n}(f) \mathrm{e}^{\mathrm{i} p \cdot x}=\frac{f^{n}}{n !}|p|^{n+1} \mathrm{e}^{\mathrm{i} p \cdot x}-\left(\nabla_{x} f\right) & \frac{f^{n-1}}{(n-1) !} \cdot(\mathrm{i} p)|p|^{n-1} \mathrm{e}^{\mathrm{i} p \cdot x} \\
& -\sum_{l=0}^{n-1} G_{l}(f)\left[\frac{f^{n-l}}{(n-l) !}|p|^{n-l} \mathrm{e}^{\mathrm{i} p \cdot x}\right] .
\end{aligned}
$$

Setting $D=-\mathrm{i} \nabla_{x}$, we derive from (2.9) the recursion

$$
G_{n}(f) \xi(x)=D \frac{f^{n}}{n !} D|D|^{n-1} \xi(x)-\sum_{l=0}^{n-1} G_{l}(f)\left[\frac{f^{n-l}}{(n-l) !}|D|^{n-l} \xi(x)\right] .
$$


Finally, using the self-adjoint nature of $G_{n}$ and $|D|$, we may rewrite $(2.10)$ in the form

$$
G_{n}(f) \xi(x)=|D|^{n-1} D \frac{f^{n}}{n !} D \xi(x)-\sum_{l=0}^{n-1}|D|^{n-l} \frac{f^{n-l}}{(n-l) !} G_{l}(f) \xi(x)
$$

(see [11]). As mentioned in the $\S 1$, these formulae were used in $[10,11,20,21]$ as a basis for a numerical algorithm for the simulation of gravity water waves. As in (2.7), the high-order derivatives that are present in (2.11) demand the smoothness of the profile $y=f(x)$ to guarantee that each individual term in the right-hand side belongs, for instance, to the space $L^{2}$. The theoretical results quoted above, on the other hand, do imply that the overall sum in (2.11) is well defined for rather rough profiles, so that substantial cancellation must take place. This, in turn, suggests that a straightforward numerical implementation of high-order versions of (2.6) or (2.11) could face severe impediments.

\section{Cancellations}

To illustrate the rather dramatic effects of the cancellations in (2.6) and (2.11), we shall consider specific instances of these for analytic perturbations of a half-plane in two dimensions of periodicity $2 \pi$. More precisely, we shall take

$$
\begin{aligned}
& f(x)=2 \cos (x)=\mathrm{e}^{\mathrm{i} x}+\mathrm{e}^{-\mathrm{i} x}, \\
& \xi(x)=2 \cos (x) .
\end{aligned}
$$

To motivate our discussion (and the subsequent analysis of $\S \S 4,5$ ), let us consider first the system (2.6). If $f$ is smooth, classical elliptic estimates [18] imply, for instance, that

$$
\begin{aligned}
& \left\|v_{n}\right\|_{H^{s}([0,2 \pi] \times[0,-\infty))} \\
& \quad \leqslant C\left\|H_{n}\right\|_{H^{s-1 / 2}([0,2 \pi])} \\
& \quad \leqslant C \sum_{l=0}^{n-1}\left\|\frac{f^{n-l}}{(n-l) !} \partial_{y}^{n-l} v_{l}(x, 0)\right\|_{H^{s-1 / 2}([0,2 \pi])}+\delta_{n, 0}\|\xi(x)\|_{H^{s-1 / 2}([0,2 \pi])} .
\end{aligned}
$$

Thus an alternative approach to a proof of analyticity suggests itself, namely the recursive estimation of $v_{n}$ and its spatial derivatives. An attempt at this procedure, however, reveals its implausibility, as the second bound in (3.2) destroys the cancellations even in the case of analytic boundaries. To see this, consider the spectral representation of the solution of (2.6),

$$
v_{n}(x, y)=\sum_{k=-\infty}^{\infty} d_{n, k} \mathrm{e}^{\mathrm{i} k x+|k| y}
$$

Then $(2.6 b)$ translates into a recursion for the Fourier coefficients $d_{n, k}$,

$$
d_{n, k}=-\sum_{l=0}^{n-1} \sum_{q=-l-1}^{l+1} C_{n-l, k-q} d_{l, q}|q|^{n-l}+\delta_{n, 0} \hat{\xi}(k)
$$


for $|k| \leqslant n+1$, where we have set

$$
\begin{aligned}
\frac{f(x)^{l}}{l !} & =\sum_{s=-l}^{l} C_{l, s} \mathrm{e}^{\mathrm{i} s x}, \\
\xi(x) & =\sum_{k=-\infty}^{\infty} \hat{\xi}(k) \mathrm{e}^{\mathrm{i} k x},
\end{aligned}
$$

that is,

$$
\begin{aligned}
C_{l, s} & = \begin{cases}\frac{1}{((l-s) / 2) !((l+s) / 2) !} & \text { if } l+s \text { is even, } \\
0 & \text { if } l+s \text { is odd }\end{cases} \\
\hat{\xi}(k) & = \begin{cases}1 & \text { if } k= \pm 1, \\
0 & \text { otherwise. }\end{cases}
\end{aligned}
$$

The convergence of the expansion (2.5) for $y=0$ in $L^{2}$ (see, for example, [3]) implies that

$$
\sum_{n=0}^{\infty}\left\|v_{n}(x, 0)\right\|_{L^{2}}^{2} \varepsilon^{n}=\sum_{n=0}^{\infty} \sum_{k=-n-1}^{n+1}\left|d_{n, k}\right|^{2} \varepsilon^{n}<\infty
$$

for $\varepsilon$ sufficiently small. In particular, letting

$$
P_{n}=d_{n, n+1},
$$

equation (3.5) implies that

$$
\sum_{n=0}^{\infty}\left|P_{n}\right| \varepsilon^{n}<\infty
$$

for small values of $\varepsilon$ and, from (3.3),

$$
P_{n}=-\sum_{l=0}^{n-1} \frac{(l+1)^{n-l}}{(n-l) !} P_{l}+\delta_{n, 0} \hat{\xi}(1) .
$$

The effect of the cancellations can now be easily illustrated by considering the majorizing sequence

$$
\Theta_{n}=\sum_{l=0}^{n-1} \frac{(l+1)^{n-l}}{(n-l) !} \Theta_{l}+\delta_{n, 0} \hat{\xi}(1),
$$

which satisfies $\left|P_{n}\right| \leqslant \Theta_{n}$ (compare (3.2)). Indeed, it can be shown [14] that while the series (3.6) converges for $\varepsilon<\mathrm{e}^{-1}$, the series $\sum_{n=0}^{\infty} \Theta_{n} \varepsilon^{n}$ diverges for all $\varepsilon \neq 0$ (see figure 1).

A similar situation holds for the recursion (2.11). In this case, again for $f$ and $\xi$ as in (3.1), we have

$$
\begin{aligned}
G_{0} \xi(x) & =2 \cos (x), \\
G_{1}(f) \xi(x) & =0, \\
G_{2}(f) \xi(x) & =-2 \cos (x) .
\end{aligned}
$$




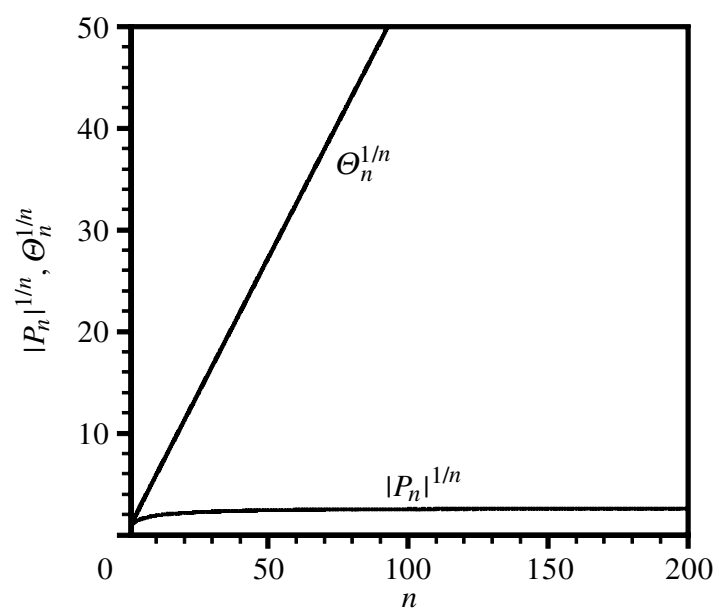

Figure 1. Plot of the numerical values of a convergence test for $P_{n}$ and $\Theta_{n}$ (cf. (3.7), (3.8)).

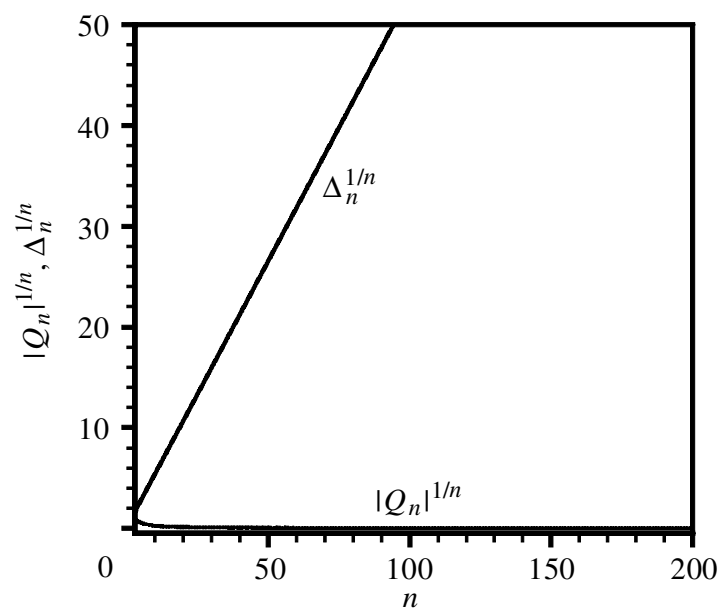

Figure 2. Plot of the numerical values of a convergence test for $Q_{n}$ and $\Delta_{n}$ (cf. (3.10), (3.11)).

In general, letting

$$
G_{n}(f) \xi=\sum_{k=-n-1}^{n+1} b_{n, k} \mathrm{e}^{\mathrm{i} k x}
$$

equation (2.11) implies

$$
\begin{array}{rl}
\sum_{k=-n-1}^{n+1} b_{n, k} \mathrm{e}^{\mathrm{i} k x}=|D|^{n-1} & D\left(\sum_{k=-n}^{n} C_{n, k} \mathrm{e}^{\mathrm{i} k x}\right)\left(\sum_{k=-1}^{1} k \hat{\xi}(k) \mathrm{e}^{\mathrm{i} k x}\right) \\
& -\sum_{l=0}^{n-1}|D|^{n-l}\left(\sum_{k=-n+l}^{n-l} C_{n-l, k} \mathrm{e}^{\mathrm{i} k x}\right)\left(\sum_{k=-l-1}^{l+1} b_{l, k} \mathrm{e}^{\mathrm{i} k x}\right) .
\end{array}
$$




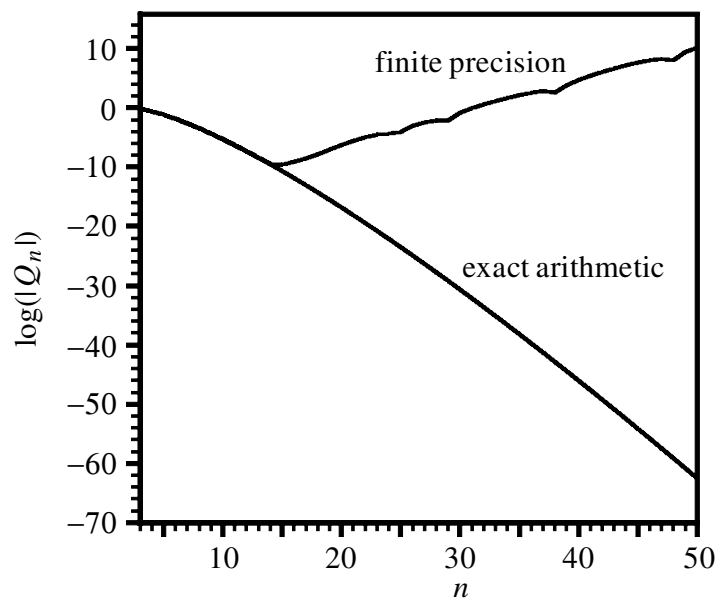

Figure 3. Plot of $\log \left(\left|Q_{n}\right|\right)$ versus $n$ in finite precision and exact arithmetic.

Using (3.4), we obtain

$b_{n, k}=|k|^{n-1} k \sum_{q=\max \{-1, k-n\}}^{\min \{1, k+n\}} C_{n, k-q} q \hat{\xi}(q)-\sum_{l=0}^{n-1}|k|^{n-l} \sum_{q=\max \{-l-1, k-n+l\}}^{\min \{l+1, k+n-l\}} C_{n-l, k-q} b_{l, q}$.

Now, from (3.9), we have $b_{0, k}=\delta_{|k|, 1}, b_{1, k}=0$ and $b_{2, k}=-\delta_{|k|, 1}$, and letting

$$
Q_{n}=b_{n, n-1} \text {, }
$$

a straightforward inductive argument shows that

$$
Q_{n}=-2 \frac{(n-1)^{n}}{n !}-\sum_{l=2}^{n-1} \frac{(n-1)^{n-l}}{(n-l) !} Q_{l}
$$

for $n \geqslant 3$. Again, as before, we have plotted in figure 2 the values of $\left|Q_{n}\right|^{1 / n}$ and those corresponding to the majorizing sequence

$$
\Delta_{n}=2 \frac{(n-1)^{n}}{n !}+\sum_{l=2}^{n-1} \frac{(n-1)^{n-l}}{(n-l) !} \Delta_{l}
$$

In this case, we have computed, in addition, the values of $Q_{n}$, as derived from (3.10), using both finite double precision and exact arithmetic (using MATLAB and MAPLE, respectively). The results showing the detrimental effect of the cancellations are presented in figure 3 and table 1.

\section{Change of variables}

In our discussion of $\S 3$, we alluded to a possible alternative proof of analyticity of DNOs that is suggested by the recursive relations (2.6), and we showed that such a procedure could only succeed if cancellations are accounted for properly. In this and the next sections we shall show that this can be achieved through a change of variables. 
Table 1. Comparison of $Q_{n}$ in finite double precision versus exact arithmetic

\begin{tabular}{rllc}
\hline & \multicolumn{1}{c}{$Q_{n}$ (finite precision) } & \multicolumn{1}{c}{$Q_{n}$ (exact arithmetic) } & $\begin{array}{c}\text { digits of } \\
\text { accuracy }\end{array}$ \\
\hline 4 & -0.25 & -0.25 & 16 \\
5 & -0.06666666666666643 & -0.06666666666666667 & 14 \\
6 & -0.01388888888889284 & -0.01388888888888889 & 12 \\
7 & -0.002380952380960366 & -0.002380952380952380 & 11 \\
8 & -0.0003472222221034826 & -0.0003472222222222222 & 9 \\
9 & $-4.409171117458754 \times 10^{-5}$ & $-4.409171075837742 \times 10^{-5}$ & 7 \\
10 & $-4.960316573487944 \times 10^{-6}$ & $-4.960317460317460 \times 10^{-6}$ & 6 \\
11 & $-5.010433596908115 \times 10^{-7}$ & $-5.010421677088343 \times 10^{-7}$ & 5 \\
12 & $-4.592948243953288 \times 10^{-8}$ & $-4.592886537330981 \times 10^{-8}$ & 4 \\
13 & $-3.841705620288849 \times 10^{-9}$ & $-3.854170520837187 \times 10^{-9}$ & 2 \\
14 & $-3.637978807091713 \times 10^{-10}$ & $-2.982393855409728 \times 10^{-10}$ & 0 \\
15 & $2.3283064365386960 \times 10^{-10}$ & $-2.141205844909548 \times 10^{-11}$ & 0 \\
\hline
\end{tabular}

For the present configuration, we introduce the transformation

$$
x^{\prime}=x, \quad y^{\prime}=\frac{y-\varepsilon f(x)}{1+\varepsilon f(x)},
$$

mapping the domain $S_{\varepsilon f}$ onto the strip $S_{0}=P(\Gamma) \times[-1,0]$. The original problem (2.2) (with $\sigma=\varepsilon f$ ) then transforms into

$$
\begin{aligned}
\Delta^{\prime} u & =F\left(x^{\prime}, y^{\prime}, \varepsilon\right) \quad \text { in } S_{0}, \\
u\left(x^{\prime}, 0, \varepsilon\right) & =\xi\left(x^{\prime}\right), \\
\partial_{y^{\prime}} u\left(x^{\prime},-1\right) & =0, \\
u\left(x^{\prime}+\gamma, y^{\prime}\right) & =u\left(x^{\prime}, y^{\prime}\right) \quad \text { for all } \gamma \in \Gamma,
\end{aligned}
$$

where

$$
u\left(x^{\prime}, y^{\prime}, \varepsilon\right)=v\left(x^{\prime}, y^{\prime}+\varepsilon f\left(x^{\prime}\right)\left(1+y^{\prime}\right), \varepsilon\right)
$$

and

$$
\begin{gathered}
F\left(x^{\prime}, y^{\prime}, \varepsilon\right)=\operatorname{div}_{x^{\prime}}\left[\frac{\varepsilon\left(\nabla_{x^{\prime}} f\right)\left(1+y^{\prime}\right)}{1+\varepsilon f} \partial_{y^{\prime}} u\right] \\
+\partial_{y^{\prime}}\left[\frac{\varepsilon\left(1+y^{\prime}\right)}{1+\varepsilon f} \nabla_{x^{\prime}} f \cdot \nabla_{x^{\prime}} u\right. \\
\left.\quad-\frac{\varepsilon^{2}\left|\nabla_{x^{\prime}} f\right|^{2}\left(1+y^{\prime}\right)^{2}}{(1+\varepsilon f)^{2}} \partial_{y^{\prime}} u-\frac{1}{(1+\varepsilon f)^{2}} \partial_{y^{\prime}} u+\partial_{y^{\prime}} u\right] \\
-\frac{\varepsilon}{1+\varepsilon f} \nabla_{x^{\prime}} f \cdot \nabla_{x^{\prime}} u+\frac{\varepsilon^{2}\left|\nabla_{x^{\prime}} f\right|^{2}\left(1+y^{\prime}\right)}{(1+\varepsilon f)^{2}} \partial_{y^{\prime}} u .
\end{gathered}
$$

In these new coordinates, and upon dropping the primes, the Dirichlet-Neumann operator takes the form

$$
\begin{aligned}
G(\varepsilon f) \xi & =\left.\left(\nabla v \cdot N_{\varepsilon f}\right)\right|_{y=\varepsilon f} \\
& =-\varepsilon \nabla_{x} f \cdot \nabla_{x} u(x, 0, \varepsilon)+\frac{1+\varepsilon^{2}\left|\nabla_{x} f\right|^{2}}{1+\varepsilon f} \partial_{y} u(x, 0, \varepsilon) .
\end{aligned}
$$


Since $\nabla_{x} u(x, 0, \varepsilon)=\nabla_{x} \xi(x)$, the evaluation of (4.2) reduces to that of $\partial_{y} u(x, 0, \varepsilon)$. For this, we formally expand

$$
u(x, y, \varepsilon)=\sum_{n=0}^{\infty} u_{n}(x, y) \varepsilon^{n},
$$

so that

$$
\partial_{y} u(x, y, \varepsilon)=\sum_{n=0}^{\infty} \partial_{y} u_{n}(x, y) \varepsilon^{n},
$$

and proceed to derive equations for the functions $u_{n}$. At order zero we have

$$
\begin{array}{rlrl}
\Delta u_{0} & =0 & & \text { in } S_{0}, \\
u_{0}(x, 0) & =\xi(x), & & \\
\partial_{y} u_{0}(x,-1) & =0, & \\
u_{0}(x+\gamma, y) & =u_{0}(x, y) \quad & \text { for all } \gamma \in \Gamma,
\end{array}
$$

and for $n>0$,

$$
\begin{aligned}
\Delta u_{n} & =F_{n}(x, y) \quad \text { in } S_{0}, \\
u_{n}(x, 0) & =0, \\
\partial_{y} u_{n}(x,-1) & =0, \\
u_{n}(x+\gamma, y) & =u_{n}(x, y) \quad \text { for all } \gamma \in \Gamma,
\end{aligned}
$$

where

$$
F_{n}(x, y)=\operatorname{div}_{x}\left[F_{n}^{(1)}(x, y)\right]+\partial_{y} F_{n}^{(2)}(x, y)+F_{n}^{(3)}(x, y)
$$

and

$$
\begin{aligned}
F_{n}^{(1)}(x, y)=(1+y)\left(\nabla_{x} f\right) \sum_{l=0}^{n-1}(-f)^{l} \partial_{y} u_{n-1-l}, \\
F_{n}^{(2)}(x, y)=(1+y)\left(\nabla_{x} f\right) \cdot \sum_{l=0}^{n-1}(-f)^{l} \nabla_{x} u_{n-1-l} \\
-(1+y)^{2}\left|\nabla_{x} f\right|^{2} \sum_{l=0}^{n-2}(l+1)(-f)^{l} \partial_{y} u_{n-2-l} \\
+f \sum_{l=0}^{n-1}(l+2)(-f)^{l} \partial_{y} u_{n-1-l}, \\
F_{n}^{(3)}(x, y)=-\left(\nabla_{x} f\right) \cdot \sum_{l=0}^{n-1}(-f)^{l} \nabla_{x} u_{n-1-l} \\
+(1+y)\left|\nabla_{x} f\right|^{2} \sum_{l=0}^{n-2}(l+1)(-f)^{l} \partial_{y} u_{n-2-l} .
\end{aligned}
$$

As will be apparent from our discussion below, and in contrast with the formulae for $H_{n}$ in (2.7), the representation of $F_{n}$ in (4.7) and (4.8) does not involve strong 
cancellations. Indeed, we shall show that the functions $u_{n}$ can be explicitly and recursively estimated in a manner that guarantees the convergence of the series in (4.3) and (4.4).

\section{Analyticity}

In order to state our results precisely, let us initially introduce our notation for the function spaces of relevance. First recall that any function $\mu \in L^{2}(P(\Gamma))$ can be expressed as

$$
\mu(x)=\frac{1}{\sqrt{2 \pi}^{n}} \sum_{k \in \Gamma^{\prime}} \hat{\mu}(k) \mathrm{e}^{\mathrm{i} k \cdot x},
$$

where

$$
\hat{\mu}(k)=\frac{1}{\sqrt{2 \pi}^{n}} \int_{P(\Gamma)} \mu(x) \mathrm{e}^{-\mathrm{i} k \cdot x} \mathrm{~d} x
$$

and $\sum_{k \in \Gamma^{\prime}}|\hat{\mu}(k)|^{2}<\infty$. We define the symbol $\langle k\rangle$ as

$$
\langle k\rangle=\sqrt{1+|k|^{2}}
$$

and use this to introduce the following Sobolev norms,

$$
\begin{aligned}
\|\mu\|_{H^{s}(P(\Gamma))}^{2} & =\sum_{k \in \Gamma^{\prime}}|\hat{\mu}(k)|^{2}\langle k\rangle^{2 s}, \\
\|\nu\|_{H^{s}([-1,0])}^{2} & =\sum_{l=0}^{s} \int_{-1}^{0}\left|\partial_{y}^{l} \nu(y)\right|^{2} \mathrm{~d} y, \\
\|w\|_{H^{s}(P(\Gamma) \times[-1,0])}^{2} & =\sum_{l=0}^{s} \sum_{k \in \Gamma^{\prime}} \int_{-1}^{0}\left|\partial_{y}^{l} \hat{w}(k, y)\right|^{2} \mathrm{~d} y\langle k\rangle^{2(s-l)},
\end{aligned}
$$

which define the obvious Sobolev spaces. $C^{s}(P(\Gamma))$ is the space of continuous functions $\mu: P(\Gamma) \rightarrow \mathbb{R}$ with $s$ many continuous derivatives. If $s$ is not an integer, then these refer to the classical Hölder spaces. Finally, we note that for positive integers $s$,

$$
\|f \mu\|_{H^{s}(P(\Gamma))} \leqslant C(s)|f|_{C^{s}}\|\mu\|_{H^{s}(P(\Gamma))},
$$

and furthermore, as we establish in lemma A.1, that given $\delta>0$,

$$
\|f \mu\|_{H^{s+1 / 2}(P(\Gamma))} \leqslant \bar{C}(s)|f|_{C^{s+1 / 2+\delta}}\|\mu\|_{H^{s+1 / 2}(P(\Gamma))} .
$$

We now state the main theorem, followed by the essential lemmas necessary to prove it. Finally, we close with the proof of the theorem itself.

Theorem 5.1. Given an integer $s \geqslant 0$ and any $\delta>0$, if $f \in C^{s+3 / 2+\delta}(P(\Gamma))$ and $\xi \in H^{s+1}(P(\Gamma))$, there exists a unique solution,

$$
u(x, y, \varepsilon)=\sum_{n=0}^{\infty} u_{n}(x, y) \varepsilon^{n},
$$


of (4.1) satisfying

$$
\begin{aligned}
\left\|\partial_{y} u_{n}(x, 0)\right\|_{H^{s}(P(\Gamma))} & \leqslant K_{0}\|\xi\|_{H^{s+1}(P(\Gamma))} B^{n}, \\
\sum_{k \in \Gamma^{\prime}}\langle k\rangle^{2 s+1}\left\|\partial_{y} \hat{u}_{n}(k, y)\right\|_{L^{2}([-1,0])(\mathrm{d} y)}^{2} & \leqslant K_{0}^{2}\|\xi\|_{H^{s+1}(P(\Gamma))}^{2} B^{2 n}, \\
\sum_{k \in \Gamma^{\prime}}\langle k\rangle^{2 s+3}\left\|\hat{u}_{n}(k, y)\right\|_{L^{2}([-1,0])(\mathrm{d} y)}^{2} & \leqslant K_{0}^{2}\|\xi\|_{H^{s+1}(P(\Gamma))}^{2} B^{2 n}
\end{aligned}
$$

for any $B>C_{0}(s)|f|_{C^{s+3 / 2+\delta}}$, where $K_{0}$ is a universal constant and $C_{0}(s)$ depends only on $s$.

To establish (5.4), we shall use estimates for general problems of the form (4.5) and (4.6), along the lines of classical elliptic regularity theory [18], and which, for the sake of completeness, we explicitly derive in lemmas 5.2 and 5.3 below. To deal with (4.5) then, we shall resort to the following lemma.

LEMMA 5.2. Given an integer $s \geqslant 0$, if $\xi \in H^{s+1}(P(\Gamma))$, then there exists a unique solution $w(x, y)$ of

$$
\begin{array}{rlrl}
\Delta w & =0 & & \text { in } S_{0}, \\
w(x, 0) & =\xi(x), & & \\
\partial_{y} w(x,-1) & =0, & \\
w(x+\gamma, y) & =w(x, y) \quad \text { for all } \gamma \in \Gamma
\end{array}
$$

satisfying

$$
\begin{array}{r}
\left\|\partial_{y} w(x, 0)\right\|_{H^{s}(P(\Gamma))} \leqslant K_{0}\|\xi\|_{H^{s+1}(P(\Gamma))}, \\
\sum_{k \in \Gamma^{\prime}}\langle k\rangle^{2 s+1}\left\|\partial_{y} \hat{w}(k, y)\right\|_{L^{2}([-1,0])(\mathrm{d} y)}^{2} \leqslant K_{0}^{2}\|\xi\|_{H^{s+1}(P(\Gamma))}^{2}, \\
\sum_{k \in \Gamma^{\prime}}\langle k\rangle^{2 s+3}\|\hat{w}(k, y)\|_{L^{2}([-1,0])(\mathrm{d} y)}^{2} \leqslant K_{0}^{2}\|\xi\|_{H^{s+1}(P(\Gamma))}^{2},
\end{array}
$$

where $K_{0}$ is a universal constant.

Proof. The Fourier transform of the solution of (5.5) can be written as

$$
\hat{w}(k, y)=\hat{\xi}(k) \frac{\cosh (|k|(y+1))}{\cosh (|k|)},
$$

and its $y$ derivative as

$$
\partial_{y} \hat{w}(k, y)=\hat{\xi}(k)|k| \frac{\sinh (|k|(y+1))}{\cosh (|k|)}
$$


for all $k \in \Gamma^{\prime}$. The first estimate (5.6a) results from evaluating (5.7) at $y=0$ and noting that $(|k| \tanh (|k|))$ is bounded by $\langle k\rangle$. To establish $(5.6 b)$, we compute

$$
\begin{aligned}
\sum_{k \in \Gamma^{\prime}}\langle k\rangle^{2 s+1} & \left\|\partial_{y} \hat{w}(x, y)\right\|_{L^{2}([-1,0])(\mathrm{d} y)}^{2} \\
& =\sum_{k \in \Gamma^{\prime}}\langle k\rangle^{2 s+1} \int_{-1}^{0}|k|^{2} \frac{\sinh ^{2}(|k|(y+1))}{\cosh ^{2}(|k|)}|\hat{\xi}(k)|^{2} \mathrm{~d} y \\
& =\sum_{k \in \Gamma^{\prime} \backslash\{0\}} \frac{\langle k\rangle^{2 s+1}|k|^{2}}{\cosh ^{2}(|k|)}\left[-\frac{1}{2}+\frac{\sinh (2|k|)}{4|k|}\right]|\hat{\xi}(k)|^{2} \\
& \leqslant K_{0}^{2}\|\xi\|_{H^{s+1}(P(\Gamma))}^{2} .
\end{aligned}
$$

Finally, the estimate $(5.6 c)$ can be similarly derived.

To estimate the solutions $u_{n}$ for $n>0$ (cf. (4.6)), we shall use the following lemma.

LEMMA 5.3. For an integer $s \geqslant 0$, if $g^{(j)}(x, 0) \in H^{s}(P(\Gamma))$ and

$$
\sum_{k \in \Gamma^{\prime}}\langle k\rangle^{2 s+1}\left\|\hat{g}^{(j)}(k, y)\right\|_{L^{2}([-1,0])(\mathrm{d} y)}^{2}<\infty,
$$

then there exists a unique solution $w(x, y)$ of

$$
\begin{array}{rlrl}
\Delta w & =\operatorname{div}_{x}\left[g^{(1)}(x, y)\right]+\partial_{y} g^{(2)}(x, y)+g^{(3)}(x, y) & & \text { in } S_{0}, \\
w(x, 0) & =0, & & \\
\partial_{y} w(x,-1) & =0, & & \text { for all } \gamma \in \Gamma \\
w(x+\gamma, y) & =w(x, y) &
\end{array}
$$

satisfying

$$
\begin{gathered}
\sum_{k \in \Gamma^{\prime}}\langle k\rangle^{2 s+3}\|\hat{w}(k, y)\|_{L^{2}([-1,0])(\mathrm{d} y)}^{2} \leqslant K_{1}^{2} \sum_{j=1}^{3} \sum_{k \in \Gamma^{\prime}}\langle k\rangle^{2 s+1}\left\|\hat{g}^{(j)}(k, y)\right\|_{L^{2}([-1,0])(\mathrm{d} y)}^{2}, \\
\sum_{k \in \Gamma^{\prime}}\langle k\rangle^{2 s+1}\left\|\partial_{y} \hat{w}(k, y)\right\|_{L^{2}([-1,0])(\mathrm{d} y)}^{2} \leqslant K_{1}^{2} \sum_{j=1}^{3} \sum_{k \in \Gamma^{\prime}}\langle k\rangle^{2 s+1}\left\|\hat{g}^{(j)}(k, y)\right\|_{L^{2}([-1,0])(\mathrm{d} y)}^{2},
\end{gathered}
$$

and

$$
\begin{aligned}
& \left\|\partial_{y} w(x, 0)\right\|_{H^{s}(P(\Gamma))}^{2} \\
& \quad \leqslant K_{1}^{2}\left[\sum_{j=1}^{3} \sum_{k \in \Gamma^{\prime}}\langle k\rangle^{2 s+1}\left\|\hat{g}^{(j)}(k, y)\right\|_{L^{2}([-1,0])(\mathrm{d} y)}^{2}+\sum_{k \in \Gamma^{\prime}}\langle k\rangle^{2 s}\left|\hat{g}^{(j)}(k, 0)\right|^{2}\right],
\end{aligned}
$$

where $K_{1}$ is a universal constant. 
Proof. The solution of (5.8) can be written as $w=w_{1}+w_{2}+w_{3}$, where each $w_{j}$ solves (5.8), with $(5.8 a)$ replaced by

$$
\begin{aligned}
\Delta w_{1} & =\operatorname{div}_{x}\left[g^{(1)}(x, y)\right], \\
\Delta w_{2} & =\partial_{y} g^{(2)}(x, y), \\
\Delta w_{3} & =g^{(3)}(x, y),
\end{aligned}
$$

respectively. Taking the Fourier transform of these equations, we are led to solve

$$
\begin{aligned}
& \left(-|k|^{2}+\partial_{y}^{2}\right) \hat{w}_{1}(k, y)=\mathrm{i} k \cdot \hat{g}^{(1)}(k, y), \\
& \left(-|k|^{2}+\partial_{y}^{2}\right) \hat{w}_{2}(k, y)=\partial_{y} \hat{g}^{(2)}(k, y), \\
& \left(-|k|^{2}+\partial_{y}^{2}\right) \hat{w}_{3}(k, y)=\hat{g}^{(3)}(k, y),
\end{aligned}
$$

supplemented with the boundary conditions

$$
\hat{w}_{j}(k, 0)=0, \quad \partial_{y} \hat{w}_{j}(k,-1)=0 .
$$

Using the homogeneous solutions, the full solutions can be written as ('variation of parameters')

$$
\hat{w}_{j}(k, y)=A_{j}(k, y) \mathrm{e}^{|k| y}+B_{j}(k, y) \sinh (|k| y)
$$

for $k \neq 0$ and

$$
\hat{w}_{j}(0, y)=A_{j}(0, y)+B_{j}(0, y) y
$$

for $k=0$, where $A_{j}$ and $B_{j}$ can be chosen to satisfy

$$
\partial_{y} A_{j}(k, y) \mathrm{e}^{|k| y}+\partial_{y} B_{j}(k, y) \sinh (|k| y)=0
$$

for $k \neq 0$ and

$$
\partial_{y} A_{j}(0, y)+\partial_{y} B_{j}(0, y) y=0
$$

for $k=0$. Substituting this form into the differential equations leads to explicit formulae for the functions $A_{j}(k, y)$ and $B_{j}(k, y)$. For instance, in the case $j=1$, $k \neq 0$, the functions $A_{1}(k, y)$ and $B_{1}(k, y)$ satisfy

$$
\begin{aligned}
& \partial_{y} A_{1}(k, y)=-\frac{\mathrm{i} k}{|k|} \cdot \sinh (|k| y) \hat{g}^{(1)}(k, y), \\
& \partial_{y} B_{1}(k, y)=\frac{\mathrm{i} k}{|k|} \cdot \mathrm{e}^{|k| y} \hat{g}^{(1)}(k, y),
\end{aligned}
$$

which result in the solutions

$$
\begin{aligned}
& A_{1}(k, y)=\int_{y}^{0} \frac{\mathrm{i} k}{|k|} \cdot \sinh (|k| s) \hat{g}^{(1)}(k, s) \mathrm{d} s+C_{1}, \\
& B_{1}(k, y)=\int_{-1}^{y} \frac{\mathrm{i} k}{|k|} \cdot \mathrm{e}^{|k| s} \hat{g}^{(1)}(k, s) \mathrm{d} s+C_{2} .
\end{aligned}
$$

Using the boundary conditions, we can deduce that $C_{1}=0$ and that

$$
C_{2}=-\frac{\mathrm{e}^{-|k|}}{|k| \cosh (|k|)}(\mathrm{i} k) \cdot \int_{-1}^{0} \sinh (|k| s) \hat{g}^{(1)}(k, s) \mathrm{d} s .
$$


Thus, using similar reasoning, we can express the functions $w_{j}$ in the form

$$
\begin{aligned}
\hat{w}_{1}(k, y)=\frac{\sinh (|k| y)}{|k|}(\mathrm{i} k) \cdot \int_{-1}^{y} \mathrm{e}^{|k| s} \hat{g}^{(1)}(k, s) \mathrm{d} s \\
\quad+\frac{\mathrm{e}^{|k| y}}{|k|}(\mathrm{i} k) \cdot \int_{y}^{0} \sinh (|k| s) \hat{g}^{(1)}(k, s) \mathrm{d} s \\
\quad-\frac{\mathrm{e}^{-|k|} \sinh (|k| y)}{|k| \cosh (|k|)}(\mathrm{i} k) \cdot \int_{-1}^{0} \sinh (|k| s) \hat{g}^{(1)}(k, s) \mathrm{d} s,
\end{aligned}
$$

$$
\begin{aligned}
\hat{w}_{2}(k, y)=- & \sinh (|k| y) \int_{-1}^{y} \mathrm{e}^{|k| s} \hat{g}^{(2)}(k, s) \mathrm{d} s \\
& -\mathrm{e}^{|k| y} \int_{y}^{0} \cosh (|k| s) \hat{g}^{(2)}(k, s) \mathrm{d} s \\
& +\frac{\mathrm{e}^{-|k|} \sinh (|k| y)}{\cosh (|k|)} \int_{-1}^{0} \cosh (|k| s) \hat{g}^{(2)}(k, s) \mathrm{d} s,
\end{aligned}
$$

$$
\begin{aligned}
\hat{w}_{3}(k, y)= & \frac{\sinh (|k| y)}{|k|} \int_{-1}^{y} \mathrm{e}^{|k| s} \hat{g}^{(3)}(k, s) \mathrm{d} s \\
& +\frac{\mathrm{e}^{|k| y}}{|k|} \int_{y}^{0} \sinh (|k| s) \hat{g}^{(3)}(k, s) \mathrm{d} s \\
& -\frac{\mathrm{e}^{-|k|} \sinh (|k| y)}{|k| \cosh (|k|)} \int_{-1}^{0} \sinh (|k| s) \hat{g}^{(3)}(k, s) \mathrm{d} s
\end{aligned}
$$

for $k \neq 0$ and

$$
\begin{aligned}
& \hat{w}_{1}(0, y)=0, \\
& \hat{w}_{2}(0, y)=-\int_{y}^{0} \hat{g}^{(2)}(0, s) \mathrm{d} s, \\
& \hat{w}_{3}(0, y)=y \int_{-1}^{y} \hat{g}^{(3)}(0, s) \mathrm{d} s+\int_{y}^{0} s \hat{g}^{(3)}(0, s) \mathrm{d} s
\end{aligned}
$$

for $k=0$, where we have assumed, without loss of generality, that $g^{(2)}(x,-1) \equiv 0$ (see $(4.6 c)$ and $(4.8 b))$. Alternatively, we may write

$$
\begin{gathered}
\begin{array}{c}
\hat{w}_{1}(k, y)=\frac{\mathrm{i} k}{2|k|} \cdot\left[-T_{1}\left(\hat{g}^{(1)}\right)(k, y)-T_{2}\left(\hat{g}^{(1)}\right)(k, y)\right. \\
\left.\quad+T_{3}\left(\hat{g}^{(1)}\right)(k, y)+T_{4}\left(\hat{g}^{(1)}\right)(k, y)\right] \\
-\frac{\mathrm{i} k}{2|k|} \frac{\sinh (|k| y)}{\cosh (|k|)} \cdot\left[-T_{2}\left(\hat{g}^{(1)}\right)(k,-1)+T_{4}\left(\hat{g}^{(1)}\right)(k,-1)\right], \\
\hat{w}_{2}(k, y)=\frac{1}{2}\left[T_{1}\left(\hat{g}^{(2)}\right)(k, y)-T_{2}\left(\hat{g}^{(2)}\right)(k, y)-T_{3}\left(\hat{g}^{(2)}\right)(k, y)-T_{4}\left(\hat{g}^{(2)}\right)(k, y)\right] \\
+\frac{\sinh (|k| y)}{\cosh (|k|)}\left[T_{2}\left(\hat{g}^{(2)}\right)(k,-1)+T_{4}\left(\hat{g}^{(2)}\right)(k,-1)\right],
\end{array}
\end{gathered}
$$


A new approach to analyticity of Dirichlet-Neumann operators

$$
\begin{aligned}
& \hat{w}_{3}(k, y)=\frac{1}{2|k|}\left[-T_{1}\left(\hat{g}^{(3)}\right)(k, y)\right.-T_{2}\left(\hat{g}^{(3)}\right)(k, y) \\
&\left.+T_{3}\left(\hat{g}^{(3)}\right)(k, y)+T_{4}\left(\hat{g}^{(3)}\right)(k, y)\right] \\
&-\frac{1}{2|k|} \frac{\sinh (|k| y)}{\cosh (|k|)} \cdot\left[-T_{2}\left(\hat{g}^{(3)}\right)(k,-1)+T_{4}\left(\hat{g}^{(3)}\right)(k,-1)\right],
\end{aligned}
$$

where

$$
\begin{aligned}
& T_{1}(\psi)(k, y)=\int_{-1}^{y} \mathrm{e}^{-|k|(y-s)} \psi(k, s) \mathrm{d} s, \\
& T_{2}(\psi)(k, y)=\int_{y}^{0} \mathrm{e}^{-|k|(s-y)} \psi(k, s) \mathrm{d} s, \\
& T_{3}(\psi)(k, y)=\int_{-1}^{y} \mathrm{e}^{|k|(y+s)} \psi(k, s) \mathrm{d} s, \\
& T_{4}(\psi)(k, y)=\int_{y}^{0} \mathrm{e}^{|k|(y+s)} \psi(k, s) \mathrm{d} s .
\end{aligned}
$$

Now, from Schwartz's inequality, we have

$$
\begin{aligned}
& \left|T_{2}(\psi)(k,-1)\right| \leqslant \frac{1}{\sqrt{2|k|}}\left(\int_{-1}^{0}|\psi(k, s)|^{2} \mathrm{~d} s\right)^{1 / 2}, \\
& \left|T_{4}(\psi)(k,-1)\right| \leqslant \frac{\mathrm{e}^{-|k|}}{\sqrt{2|k|}}\left(\int_{-1}^{0}|\psi(k, s)|^{2} \mathrm{~d} s\right)^{1 / 2} .
\end{aligned}
$$

On the other hand,

$$
\begin{aligned}
\left(\int_{-1}^{0}\left|T_{1}(\psi)(k, y)\right|^{2} \mathrm{~d} y\right)^{1 / 2} & =\left(\int_{-1}^{0}\left|\int_{-1}^{y} \psi(k, s) \mathrm{e}^{-|k|(y-s)} \mathrm{d} s\right|^{2} \mathrm{~d} y\right)^{1 / 2} \\
& =\left(\int_{-1}^{0}\left|\int_{0}^{y+1} \psi(k, y-u) \mathrm{e}^{-|k| u} \mathrm{~d} u\right|^{2} \mathrm{~d} y\right)^{1 / 2}
\end{aligned}
$$

and using Minkowski's inequality,

$$
\begin{aligned}
\left(\int_{-1}^{0}\left|T_{1}(\psi)(k, y)\right|^{2} \mathrm{~d} y\right)^{1 / 2} & \leqslant \int_{0}^{1} \mathrm{e}^{-|k| u}\left(\int_{-1}^{0}|\psi(k, y-u)|^{2} \chi_{u<y+1} \mathrm{~d} y\right)^{1 / 2} \mathrm{~d} u \\
& \leqslant \frac{1}{|k|}\left(\int_{-1}^{0}|\psi(k, y)|^{2} \mathrm{~d} y\right)^{1 / 2}
\end{aligned}
$$

Similarly,

$$
\left(\int_{-1}^{0}\left|T_{j}(\psi)(k, y)\right|^{2} \mathrm{~d} y\right)^{1 / 2} \leqslant \frac{1}{|k|}\left(\int_{-1}^{0}|\psi(k, y)|^{2} \mathrm{~d} y\right)^{1 / 2}
$$

for $j=2,3,4$. Then it follows from (5.12), (5.13), (5.14) and (5.15) that

$$
\sum_{k \in \Gamma^{\prime}}\langle k\rangle^{2 s+3}\|\hat{w}(k, y)\|_{L^{2}([-1,0])(\mathrm{d} y)}^{2} \leqslant K_{1}^{2} \sum_{j=1}^{3} \sum_{k \in \Gamma^{\prime}}\langle k\rangle^{2 s+1}\left\|\hat{g}^{(j)}(k, y)\right\|_{L^{2}([-1,0])(\mathrm{d} y)}^{2} .
$$


Also, since

$$
\begin{aligned}
& \partial_{y} T_{1}(\psi)(k, y)=-|k| T_{1}(\psi)(k, y)+\psi(k, y), \\
& \partial_{y} T_{2}(\psi)(k, y)=|k| T_{2}(\psi)(k, y)-\psi(k, y), \\
& \partial_{y} T_{3}(\psi)(k, y)=|k| T_{3}(\psi)(k, y)+\mathrm{e}^{2|k| y} \psi(k, y), \\
& \partial_{y} T_{4}(\psi)(k, y)=|k| T_{4}(\psi)(k, y)-\mathrm{e}^{2|k| y} \psi(k, y),
\end{aligned}
$$

we deduce again from $(5.12),(5.13),(5.14)$ and $(5.15)$ that

$$
\sum_{k \in \Gamma^{\prime}}\langle k\rangle^{2 s+1}\left\|\partial_{y} \hat{w}(k, y)\right\|_{L^{2}([-1,0])(\mathrm{d} y)}^{2} \leqslant K_{1}^{2} \sum_{j=1}^{3} \sum_{k \in \Gamma^{\prime}}\langle k\rangle^{2 s+1}\left\|\hat{g}^{(j)}(k, y)\right\|_{L^{2}([-1,0])(\mathrm{d} y)}^{2} .
$$

Finally, since

$$
\begin{aligned}
& \left|T_{1}(\psi)(k, 0)\right| \leqslant \frac{1}{\sqrt{2|k|}}\left(\int_{-1}^{0}|\psi(k, y)|^{2} \mathrm{~d} y\right)^{1 / 2}, \\
& \left|T_{3}(\psi)(k, 0)\right| \leqslant \frac{1}{\sqrt{2|k|}}\left(\int_{-1}^{0}|\psi(k, y)|^{2} \mathrm{~d} y\right)^{1 / 2},
\end{aligned}
$$

the estimate $(5.9 c)$ follows from $(5.12),(5.16)$ and $(5.17)$.

The final lemma relates to the recursive estimation of the functions $F_{n}$ in $(4.7)$ and (4.8).

LEMMA 5.4. Let $s \geqslant 0$ be an integer and let $f \in C^{s+3 / 2+\delta}(P(\Gamma))$ for some $\delta>0$. Assume

$$
\begin{aligned}
\left\|\nabla_{x} u_{m}(x, 0)\right\|_{H^{s}(P(\Gamma))} & \leqslant K_{2} B^{m}, \\
\left\|\partial_{y} u_{m}(x, 0)\right\|_{H^{s}(P(\Gamma))} & \leqslant K_{2} B^{m}, \\
\sum_{k \in \Gamma^{\prime}}\langle k\rangle^{2 s+1}\left\|\partial_{y} \hat{u}_{m}(k, y)\right\|_{L^{2}([-1,0])(\mathrm{d} y)}^{2} & \leqslant K_{2}^{2} B^{2 m}, \\
\sum_{k \in \Gamma^{\prime}}\langle k\rangle^{2 s+3}\left\|\hat{u}_{m}(k, y)\right\|_{L^{2}([-1,0])(\mathrm{d} y)}^{2} & \leqslant K_{2}^{2} B^{2 m}
\end{aligned}
$$

for constants $K_{2}, B>0$ and for all $m<n$. Then if

$$
B>2 \max \left\{C(s)|f|_{C^{s+1}}, \bar{C}(s)|f|_{C^{s+3 / 2+\delta}}\right\},
$$

the functions $F_{n}^{(j)}$ in (4.8) satisfy

$$
\begin{aligned}
\left\|F_{n}^{(j)}(x, 0)\right\|_{H^{s}(P(\Gamma))} & \leqslant K_{3} C(s)|f|_{C^{s+1}} K_{2} B^{n-1}, \\
\sum_{k \in \Gamma^{\prime}}\langle k\rangle^{2 s+1}\left\|\hat{F}_{n}^{(j)}(k, y)\right\|_{L^{2}([-1,0])(\mathrm{d} y)}^{2} & \leqslant K_{3}^{2} \bar{C}(s)^{2}|f|_{C^{s+3 / 2+\delta}}^{2} K_{2}^{2} B^{2(n-1)}
\end{aligned}
$$

for a universal constant $K_{3}$.

Proof. Recalling (5.2) and (5.3), we set, for convenience,

$$
\alpha=C(s)|f|_{C^{s}}, \quad \beta=\bar{C}(s)|f|_{C^{s+1 / 2+\delta}} .
$$


We will prove the estimates for the functions $F_{n}^{(2)}$; the estimates for $F_{n}^{(1)}$ and $F_{n}^{(3)}$ can be established in a similar manner. From (4.8) and (5.18), we have

$$
\begin{aligned}
\left\|F_{n}^{(2)}(x, 0)\right\|_{H^{s}(P(\Gamma))} \leqslant & C(s)\left|\nabla_{x} f\right|_{C^{s}} K_{2} B^{n-1} \sum_{l=0}^{n-1}\left(\frac{\alpha}{B}\right)^{l} \\
& +C(s)^{2}\left|\nabla_{x} f\right|_{C^{s}}^{2} K_{2} B^{n-2} \sum_{l=0}^{n-2}(l+1)\left(\frac{\alpha}{B}\right)^{l} \\
& +C(s)|f|_{C^{s}} K_{2} B^{n-1} \sum_{l=0}^{n-1}(l+2)\left(\frac{\alpha}{B}\right)^{l} \\
\leqslant & 16 C(s)|f|_{C^{s+1}} K_{2} B^{n-1},
\end{aligned}
$$

where we have used $B>C(s)\left|\nabla_{x} f\right|_{C^{s}}$ and $B>2 \alpha$. Finally, to derive (5.19b), we compute

$$
\begin{aligned}
\sum_{k \in \Gamma^{\prime}}\langle k\rangle^{2 s+1}\left\|\hat{F}_{n}^{(2)}(k, y)\right\|_{L^{2}([-1,0])(\mathrm{d} y)}^{2} & \int_{-1}^{0}\left\|F_{n}^{(2)}(x, y)\right\|_{H^{s+1 / 2}(P(\Gamma))}^{2} \mathrm{~d} y \\
\leqslant & \bar{C}(s)^{2}\left|\nabla_{x} f\right|_{C^{s+1 / 2+\delta}}^{2} K_{2}^{2} B^{2(n-1)}\left(\sum_{l=0}^{n-1}\left(\frac{\beta}{B}\right)^{l}\right)^{2} \\
& +\bar{C}(s)^{4}\left|\nabla_{x} f\right|_{C^{s+1 / 2+\delta}}^{4} K_{2}^{2} B^{2(n-2)}\left(\sum_{l=0}^{n-2}(l+1)\left(\frac{\beta}{B}\right)^{l}\right)^{2} \\
& +\bar{C}(s)^{2}|f|_{C^{s+1 / 2+\delta}}^{2} K_{2}^{2} B^{2(n-1)}\left(\sum_{l=0}^{n-1}(l+2)\left(\frac{\beta}{B}\right)^{l}\right)^{2} \\
\leqslant & 56 \bar{C}(s)^{2}|f|_{C^{s+3 / 2+\delta}}^{2} K_{2}^{2} B^{2(n-1)},
\end{aligned}
$$

where we have used $B>\bar{C}(s)\left|\nabla_{x} f\right|_{C^{s+1 / 2+\delta}}$ and $B>2 \beta$.

Proof of theorem 5.1. We will prove the estimates of (5.4) inductively. The case $n=0$ follows immediately from lemma 5.2. We now assume estimates (5.4) hold for all $n<M$ and we set

$$
K_{2}=K_{0}\|\xi\|_{H^{s+1}(P(\Gamma))} .
$$

Then, from lemma 5.3,

$$
\begin{gathered}
\sum_{k \in \Gamma^{\prime}}\langle k\rangle^{2 s+3}\left\|\hat{u}_{M}(k, y)\right\|_{L^{2}([-1,0])(\mathrm{d} y)}^{2} \leqslant K_{1}^{2} \sum_{j=1}^{3} \sum_{k \in \Gamma^{\prime}}\langle k\rangle^{2 s+1}\left\|\hat{F}_{M}^{(j)}(k, y)\right\|_{L^{2}([-1,0])(\mathrm{d} y)}^{2}, \\
\sum_{k \in \Gamma^{\prime}}\langle k\rangle^{2 s+1}\left\|\partial_{y} \hat{u}_{M}(k, y)\right\|_{L^{2}([-1,0])(\mathrm{d} y)}^{2} \\
\leqslant K_{1}^{2} \sum_{j=1}^{3} \sum_{k \in \Gamma^{\prime}}\langle k\rangle^{2 s+1}\left\|\hat{F}_{M}^{(j)}(k, y)\right\|_{L^{2}([-1,0])(\mathrm{d} y)}^{2},
\end{gathered}
$$


and

$\left\|\partial_{y} u_{M}(x, 0)\right\|_{H^{s}(P(\Gamma))}^{2}$

$$
\leqslant K_{1}^{2}\left[\sum_{j=1}^{3} \sum_{k \in \Gamma^{\prime}}\langle k\rangle^{2 s+1}\left\|\hat{F}_{M}^{(j)}(k, y)\right\|_{L^{2}([-1,0])(\mathrm{d} y)}^{2}+\sum_{k \in \Gamma^{\prime}}\langle k\rangle^{2 s}\left|\hat{F}_{M}^{(j)}(k, 0)\right|^{2}\right],
$$

and, using lemma 5.4, we obtain

$$
\begin{aligned}
\sum_{k \in \Gamma^{\prime}}\langle k\rangle^{2 s+3}\left\|\hat{u}_{M}(k, y)\right\|_{L^{2}([-1,0])(\mathrm{d} y)}^{2} & \leqslant 3 K_{1}^{2} K_{2}^{2} K_{3}^{2} \bar{C}(s)^{2}|f|_{C^{s+3 / 2+\delta}}^{2} B^{2(M-1)} \\
\sum_{k \in \Gamma^{\prime}}\langle k\rangle^{2 s+1}\left\|\partial_{y} \hat{u}_{M}(k, y)\right\|_{L^{2}([-1,0])(\mathrm{d} y)}^{2} & \leqslant 3 K_{1}^{2} K_{2}^{2} K_{3}^{2} \bar{C}(s)^{2}|f|_{C^{s+3 / 2+\delta}}^{2} B^{2(M-1)} \\
\left\|\partial_{y} u_{M}(x, 0)\right\|_{H^{s}(P(\Gamma))}^{2} \leqslant 3 K_{1}^{2} K_{2}^{2} K_{3}^{2}\left(\bar{C}(s)^{2}|f|_{C^{s+3 / 2+\delta}}^{2}\right. & \left.+C(s)^{2}|f|_{C^{s+1}}^{2}\right) B^{2(M-1)}
\end{aligned}
$$

Finally, the estimates (5.4) for $n=M$ follow, provided we take

$$
B>\sqrt{6} K_{1} K_{3} \max \left\{\bar{C}(s)|f|_{C^{s+3 / 2+\delta}}, C(s)|f|_{C^{s+1}}\right\} .
$$

Thus we can set $C_{0}(s)=\sqrt{6} K_{1} K_{3} \max \{\bar{C}(s), C(s)\}$.

An immediate corollary of theorem 5.1 is the analyticity of DNOs. To begin, we recall (4.2) and express the DNOs as a series (cf. (2.4)),

$$
G(\varepsilon f) \xi=\sum_{n=0}^{\infty}\left(G_{n}(f) \xi\right) \varepsilon^{n}
$$

where

$G_{n}(f) \xi=\sum_{l=0}^{n}(-f)^{l} \partial_{y} u_{n-l}(x, 0)-\delta_{n, 1} \nabla_{x} f \cdot \nabla_{x} \xi(x)+\left|\nabla_{x} f\right|^{2} \sum_{l=0}^{n-2}(-f)^{l} \partial_{y} u_{n-2-l}(x, 0)$.

TheOREM 5.5. For an integer $s \geqslant 0$ and $\delta>0$, if $f \in C^{s+3 / 2+\delta}(P(\Gamma))$, then the series

$$
G(\varepsilon f)=\sum_{n=0}^{\infty} G_{n}(f) \varepsilon^{n}
$$

converges strongly as an operator from $H^{s+1}(P(\Gamma))$ to $H^{s}(P(\Gamma))$. More precisely,

$$
\left\|G_{n}(f) \xi\right\|_{H^{s}(P(\Gamma))} \leqslant\left[\frac{5}{2} K_{0}+\frac{1}{2}\right]\|\xi\|_{H^{s+1}(P(\Gamma))} B^{n}
$$

for any $B>C_{0}(s)|f|_{C^{s+3 / 2+\delta}}$, where $C_{0}(s)$ depends only on $s$. 
Proof. From (5.20), we have

$$
\begin{aligned}
\left\|G_{n}(f) \xi\right\|_{H^{s}(P(\Gamma))} \leqslant & \sum_{l=0}^{n}\left\|(-f)^{l} \partial_{y} u_{n-l}(x, 0)\right\|_{H^{s}(P(\Gamma))} \\
& +\delta_{n, 1}\left\|\nabla_{x} f \cdot \nabla_{x} \xi(x)\right\|_{H^{s}(P(\Gamma))} \\
& +\sum_{l=0}^{n-2}\left\|\left|\nabla_{x} f\right|^{2}(-f)^{l} \partial_{y} u_{n-2-l}(x, 0)\right\|_{H^{s}(P(\Gamma))} \\
\leqslant & \sum_{l=0}^{n} C(s)^{l}|f|_{C^{s}}^{l}\left\|\partial_{y} u_{n-l}(x, 0)\right\|_{H^{s}(P(\Gamma))} \\
& +\delta_{n, 1} C(s)|f|_{C^{s+1}}\left\|\nabla_{x} \xi(x)\right\|_{H^{s}(P(\Gamma))} \\
& +\sum_{l=0}^{n-2} C(s)^{2}|f|_{C^{s+1}}^{2} C(s)^{l}|f|_{C^{s}}^{l}\left\|\partial_{y} u_{n-2-l}(x, 0)\right\|_{H^{s}(P(\Gamma))} .
\end{aligned}
$$

Hence, using theorem 5.1, we can estimate

$$
\begin{aligned}
\left\|G_{n}(f) \xi\right\|_{H^{s}(P(\Gamma))} \leqslant & K_{0}\|\xi\|_{H^{s+1}(P(\Gamma))} B^{n} \sum_{l=0}^{n}\left(\frac{C(s)|f|_{C^{s}}}{B}\right)^{l} \\
& +\delta_{n, 1} C(s)|f|_{C^{s+1}}\|\xi\|_{H^{s+1}(P(\Gamma))} \\
& +K_{0}\|\xi\|_{H^{s+1}(P(\Gamma))} C(s)^{2}|f|_{C^{s+1}}^{2} B^{n-2} \sum_{l=0}^{n}\left(\frac{C(s)|f|_{C^{s}}}{B}\right)^{l} \\
\leqslant & {\left[2 K_{0}+\delta_{n, 1} \frac{1}{2}+\frac{1}{2} K_{0}\right]\|\xi\|_{H^{s+1}(P(\Gamma))} B^{n}, }
\end{aligned}
$$

since $B>2 C_{0}(s)|f|_{C^{s+3 / 2+\delta}}>2 C(s)|f|_{C^{s+1}}>2 C(s)|f|_{C^{s}}$.

REMARK 5.6. In the case of water of infinite depth $(h=\infty)$, the analogues of theorems 5.1 and 5.5 can also be established. One approach to the proof is to consider solutions of $(4.5),(4.6)$ in a space of square integrable functions weighted for exponential decay as $y \rightarrow-\infty$. An alternative method, which is also applicable to scattering problems (governed by Helmholtz's equation), involves the deformation of the integration contours in the formulae (5.10), (5.11) onto a complex ray where the solutions can be shown to decay exponentially.

\section{Acknowledgments}

F.R. gratefully acknowledges support from AFOSR through contract No. F4962099-1-0193 and from NSF through grant numbers DMS-9622555 and DMS-9971379.

\section{Acknowledgment and disclaimer}

Effort sponsored by the Air Force Office of Scientific Research, Air Force Materials Command, USAF, under grant number F49620-99-1-0193. The US Government is authorized to reproduce and distribute reprints for governmental purposes notwithstanding any copyright notation thereon. The views and conclusions contained herein are those of the authors and should not be interpreted as necessarily 
representing the official policies or endorsements, either expressed or implied, of the Air Force Office of Scientific Research or the US Government.

\section{Appendix A. A multiplier result}

Lemma A.1. Given an integer $s \geqslant 0$ and $\delta>0$, if $f \in C^{s+1 / 2+\delta}(P(\Gamma)$ ) and $\mu \in H^{s+1 / 2}(P(\Gamma))$, we have

$$
\|f \mu\|_{H^{s+1 / 2}(P(\Gamma))} \leqslant \bar{C}(s)|f|_{C^{s+1 / 2+\delta}}\|\mu\|_{H^{s+1 / 2}(P(\Gamma))}
$$

for some constant $\bar{C}(s)$ depending only on $s$.

Proof. We recall that if $s=\rho$ and $0<\rho<1$, a norm in $H^{\rho}(P(\Gamma)), P(\Gamma) \in \mathbb{R}^{N}$, which is equivalent to that in $(5.1 a)$, is given by

$$
\|\mu\|_{H^{\rho}}^{2}=\|\mu\|_{L^{2}}^{2}+\int_{P(\Gamma)} \int_{P(\Gamma)} \frac{|\mu(x)-\mu(y)|^{2}}{\left|\mathrm{e}^{\mathrm{i} x}-\mathrm{e}^{\mathrm{i} y}\right|^{2 \rho+N}} \mathrm{~d} x \mathrm{~d} y
$$

(see [22]). Now, to prove (A 1), let us begin with the case $s=0$. Using (A 2) with $\rho=\frac{1}{2}$,

$$
\begin{aligned}
\|f \mu\|_{H^{1 / 2}}^{2} & =\|f \mu\|_{L^{2}}^{2}+\int_{P(\Gamma)} \int_{P(\Gamma)} \frac{|f(x) \mu(x)-f(y) \mu(y)|^{2}}{\left|\mathrm{e}^{\mathrm{i} x}-\mathrm{e}^{\mathrm{i} y}\right|^{N+1}} \mathrm{~d} x \mathrm{~d} y \\
\leqslant & |f|_{L^{\infty}}^{2}\|\mu\|_{L^{2}}^{2}+2 \int_{P(\Gamma)} \int_{P(\Gamma)} \frac{|f(x)-f(y)|^{2}}{\left|\mathrm{e}^{\mathrm{i} x}-\mathrm{e}^{\mathrm{i} y}\right|^{N+1}}|\mu(x)|^{2} \mathrm{~d} x \mathrm{~d} y \\
& +2 \int_{P(\Gamma)} \int_{P(\Gamma)}|f(y)|^{2} \frac{|\mu(x)-\mu(y)|^{2}}{\mid \mathrm{e}^{\mathrm{i} x}-\mathrm{e}^{\mathrm{i} y \mid N+1}} \mathrm{~d} x \mathrm{~d} y .
\end{aligned}
$$

The first and third terms can be grouped together and bounded by $C|f|_{L^{\infty}}^{2}\|\mu\|_{H^{1 / 2}}^{2}$. As for the second term in (A 3), we have

$$
\begin{gathered}
\int_{P(\Gamma)} \int_{P(\Gamma)} \frac{|f(x)-f(y)|^{2}}{\left.\left|\mathrm{e}^{\mathrm{i} x}-\mathrm{e}^{\mathrm{i} y}\right|\right|^{N+1}}|\mu(x)|^{2} \mathrm{~d} x \mathrm{~d} y \\
\leqslant|f|_{C^{1 / 2+\delta}}^{2} \int_{P(\Gamma)} \int_{P(\Gamma)} \frac{|x-y|^{1+2 \delta}}{\left|\mathrm{e}^{\mathrm{i} x}-\mathrm{e}^{\mathrm{i} y}\right|^{N+1}}|\mu(x)|^{2} \mathrm{~d} x \mathrm{~d} y \\
\leqslant C|f|_{C^{1 / 2+\delta}}^{2}\|\mu\|_{L^{2}}^{2} .
\end{gathered}
$$

In general, if $s>0$,

$$
\|\mu\|_{H^{s+1 / 2}}^{2}=\|\mu\|_{H^{s}}^{2}+\left\|\nabla_{x}^{s} \mu\right\|_{H^{1 / 2}}^{2},
$$

and clearly

$$
\|f \mu\|_{H^{s}} \leqslant C(s)|f|_{C^{s}}\|\mu\|_{H^{s}} .
$$

On the other hand, the estimate

$$
\left\|\nabla_{x}^{s}(f \mu)\right\|_{H^{1 / 2}} \leqslant C(s)|f|_{C^{s+(1 / 2)+\delta}}\|\mu\|_{H^{s+1 / 2}}
$$

follows from the regularity properties of $f$ and the estimates (A 3) and (A 4). Finally, equations (A 5), (A 6) and (A 7 ) deliver (A 1) for all $s>0$. 


\section{References}

1 G. R. Barrenechea, M. A. Barrientos and G. N. Gatica. On the numerical analysis of finite element and Dirichlet-to-Neumann methods for nonlinear exterior transmission problems. Numer. Funct. Analysis Optimiz. 19 (1998), 705-735.

2 G. R. Barrenechea, G. N. Gatica and G. C. Hsiao. Weak solvability of interior transmission problems via mixed finite elements and Dirichlet-to-Neumann mappings. J. Comput. Appl. Math. 100 (1998), 145-160.

O. P. Bruno and F. Reitich. Solution of a boundary value problem for the Helmholtz equation via variation of the boundary into the complex domain. Proc. $R$. Soc. Edinb. A 122 (1992), 317-340.

4 O. P. Bruno and F. Reitich. Numerical solution of diffraction problems: a method of variation of boundaries. J. Opt. Soc. Am A 10 (1993), 1168-1175.

5 O. P. Bruno and F. Reitich. Numerical solution of diffraction problems: finitely conducting gratings, Padé approximants, and singularities. J. Opt. Soc. Am A 10 (1993), 2307-2316. O. P. Bruno and F. Reitich. Numerical solution of diffraction problems: doubly periodic gratings. J. Opt. Soc. Am A 10 (1993), 2551-2562.

A. P. Calderón. Cauchy integrals on Lipschitz curves and related operators. Proc. Natl Acad. Sci. USA 75 (1977), 1324-1327.

R. Coifman and Y. Meyer. Nonlinear harmonic analysis and analytic dependence. In Pseudodifferential operators and applications, pp. 71-78 (Providence, PA: American Mathematical Society, 1985).

9 W. Craig and D. P. Nicholls. Traveling two and three dimensional capillary gravity water waves. SIAM J. Math. Analysis 32 (2000), 323-359.

10 W. Craig and D. P. Nicholls. Traveling gravity water waves in two and three dimensions. (Preprint.)

11 W. Craig and C. Sulem. Numerical simulation of gravity waves. J. Comput. Phys. 108 (1993), 73-83.

W. Craig, U. Schanz and C. Sulem. The modulation regime of three-dimensional water waves and the Davey-Stewartson system. Annls Inst. H. Poincaré Analyse Non Linéaire 14 (1997), 615-667.

D. G. Dommermuth and D. K. P. Yue. A high-order spectral method for the study of nonlinear gravity waves. J. Fluid Mech. 184 (1987), 267-288.

A. Friedman and F. Reitich. Symmetry-breaking bifurcation of analytic solutions to free boundary problems: an application to a model of tumor growth. Trans. Am Math. Soc. 353 (2001), 1587-1634.

C. Godrèche (ed.). Solids far from equilibrium (Cambridge University Press, 1992).

M. J. Grote and J. B. Keller. Nonreflecting boundary conditions for Maxwell's equations. J. Comput. Phys. 139 (1998), 327-342.

J. B. Keller and D. Givoli. Exact nonreflecting boundary conditions. J. Comput. Phys. 82 (1989), 172-192.

18 O. A. Ladyzhenskaya and N. N. Ural'tseva. Linear and quasilinear elliptic equations (Academic, 1968).

19 H. Lamb. Hydrodynamics, 6th edn (Cambridge University Press, 1993).

20 D. P. Nicholls. Traveling water waves: spectral continuation methods with parallel implementation. J. Comput. Phys. 143 (1998), 224-240.

U. Schanz. On the evolution of gravity-capillary waves in three dimensions. PhD thesis, University of Toronto (1997).

H. Triebel. Theory of function spaces (Basel: Birkhäuser, 1983).

V. Zakharov. Stability of periodic waves of finit
J. Appl. Mech. Tech. Phys. 9 (1968), 190-194.

(Issued 14 December 2001) 